\title{
A Per-Class QoS Service Model in IEEE 802.11e WLANs
}

\author{
Jeng Farn Lee ${ }^{1,3}$, Wanjiun Liao ${ }^{1,2}$, and Meng Chang Chen ${ }^{3}$ \\ ${ }^{1}$ Department of Electrical Engineering, National Taiwan University, Taipei, Taiwan \\ ${ }^{2}$ Graduate Institute of Communication Engineering, National Taiwan University, Taipei, Taiwan \\ ${ }^{3}$ Institute of Information Science, Academia Sinica, Taipei, Taiwan
}

Abstract -- In this paper, we study the provision of perclass QoS for IEEE 802.11e Enhanced Distributed Channel Access (EDCA) WLANs. We propose two mechanisms, called BIWF-SP and IDFQ-SP, based on backoff interval (BI) and inter-frame space (IFS), respectively. In our mechanisms, both strict priority and proportional fair service are supported. We describe the operations of the proposed mechanisms in details, and compare their performance with the original EDCA mechanism via simulations. The results show that both BIWF-SP and IDFQ-SP outperform the original EDCA in terms of the support for both strict priority and weighted fair service. Compared to IDFQ$S P, B I W F-S P$ is easier to be implemented in real systems; compared to BIWF-SP, IDFQ-SP has better aggregate throughput and is more stable. More importantly, both mechanisms conform with the IEEE 802.11e EDCA standard, rendering both good candidates to provide per-class QoS service for IEEE 802.11 WLANS.

\section{Introduction}

IEEE 802.11e [3] is a supplementary standard of 802.11 to provide QoS for different kinds of applications. In 802.11e, QoS is supported with a new access method called the Hybrid Coordination Function (HCF). In HCF, two medium access mechanisms are defined. In this paper, we focus only on the HCF contention-based channel access mechanism, referred to as Enhanced Distributed Channel Access (EDCA). The EDCA of 802.11e, an enhanced version of 802.11 DCF, provides prioritybased service for different access categories (ACs). With EDCA, a station can implement up to four ACs, which are AC_VO, AC_VI, AC_BE, and AC_BK, corresponding to voice, video, best effort, and background traffic, respectively. Each $\mathrm{AC}$ is associated with one backoff entity and the operation of each backoff entity is similar to that of legacy DCF. The backoff entities are prioritized according to ACspecific parameters called the EDCA parameter set composed of Arbitrary Inter-Frame Space Number (AIFSN[AC]), minimum contention window
$(C W \min [\mathrm{AC}])$, and maximum contention window $(C W \max [\mathrm{AC}])$. The AIFSN[AC] is used to determine the duration of Arbitrary IFS (AIFS[AC]) according to

$$
\text { AIFS }[A C]=\operatorname{SIFS}+\operatorname{AIFSN}[A C] \times \text { aslotTime, }
$$

$\operatorname{AIFSN}[A C] \geq 2$

where aslotTime is the duration of a slot.

With 802.11 e EDCA, only priority-based service is supported. Such priority-based service, while allowing service differentiation for flows of different priorities, cannot ensure minimum guaranteed or expected throughputs. In addition, it cannot allow service amounts in proportion to the demands of flows. This calls for strict priority and weighted fair service to be supported by EDCA. To provide weighted fair service, fair queuing is required to be implemented in EDCA. The major challenge of fair queuing in WLANs is in that service provisioning must be fully distributed. This renders existing centralized mechanisms (e.g., [5] for wired networks and [6] for wireless networks in which base stations are involved) inappropriate. Some scheduling disciplines have been proposed for weighted fairness in the link or MAC layer of legacy 802.11 DCF, including Distributed Fair Queuing (DFS) [7], Priority-based fair Medium Access Control (P-MAC) [8], and Distributed Deficit Round Robin (DDRR) [9]. The typical approach of these works is to tune one of the three 802.11 MAC parameters, i.e., Backoff Interval (BI), Contention Window (CW), and Inter-Frame Space (IFS), based on certain fair queuing mechanisms.

The mechanisms described above all aim at providing station-based service differentiation, rather than AC-based QoS service provided by EDCA. So far, no mechanism has been designed for EDCA to provide proportional fairness service. Adaptive Fair Enhanced DCF (AFEDCF) [11] is the only mechanism that implements fairness among flows of the same priority for 802.11e EDCA. However, AFEDCF cannot provide weighted fair service among different ACs and thus is not a proportional fairness mechanism.

In this paper, we propose a differentiated service model for IEEE 802.11e WLANs. In this model, both strict priority and weighted fair service are provided by EDCA. The resource is first allocated to flows of 
higher priority to meet their delay and delay jitter requirements. The remaining bandwidth is then shared proportionally among the other service classes according to their assigned weights. We propose two mechanisms based on two 802.11 MAC parameters to implement such services for EDCA: Backoff-Interval based Weighted Fair scheduling with Strict Priority (BIWF-SP), and IFS-based Distributed Fair Queuing with Strict Priority (IDFQ-SP). The performance of both mechanisms is evaluated via simulations based on ns-2. Note that both mechanisms may also be used to provide strict priority or proportional fairness service only.

The rest of the paper is organized as follows. In Sec. II, the related work on weighted fair scheduling in 802.11 DCF is reviewed. In Sec. III, the service model and the design issues are described. Besides, the two mechanisms for differentiated service are proposed. In Sec IV, the performance of the proposed mechanisms is evaluated via simulation based on ns-2. Finally, the paper is concluded in Sec V.

\section{RELATED WORK ON WEIGHTED FAIR SCHEDULING IN 802.11 DCF}

\subsection{DFS}

DFS is designed to emulate Self-Clocked Fair Queuing (SCFQ) [5] in a distributed manner. In SCFQ, a virtual clock is maintained by the central coordinator in the system. Let $v(t)$ denote the virtual clock at real time $t, P_{i}^{k}$ be the $k^{\text {th }}$ packet of flow $i$, and $A_{i}^{k}$ be the real arrival time of $P_{i}^{k}$. Let $L_{i}^{k}$ denote the packet size of $P_{i}^{k}$ and $\phi_{i}$ the assigned weight of flow $i$. A start tag $S_{i}^{k}$ and a finish $\operatorname{tag} F_{i}^{k}$ are associated with each packet $P_{i}^{k}$, given by

$$
\begin{aligned}
S_{i}^{k} & =\max \left\{v\left(A_{i}^{k}\right), F_{i}^{k-1}\right\} \\
F_{i}^{k} & =S_{i}^{k}+\frac{L_{i}^{k}}{\phi_{i}}
\end{aligned}
$$

The system virtual clock is set to 0 initially, and is updated to the finish tag of the packet transmitted on the output link. In SCFQ, packets are transmitted in increasing order of the finish tags. Ties are broken arbitrarily.

To emulate SCFQ in which the frame with the smallest finish tag is transmitted first, each transmitted frame in DFS is stamped with a finish tag, based on which the BI for each competing station is then chosen. The backoff interval $B I_{i}$ for each mobile station $i$ is expressed as follows.

$$
B I_{i}=\left\lfloor\left\lfloor\text { Scaling_Factor } \times \frac{L_{i}}{\phi_{i}}\right\rfloor \times \rho\right\rfloor,
$$

where $L_{i}$ is the head of line frame size, $\phi_{i}$ is the assigned weight of mobile station $i$, and $\rho$ is a random variable uniformly distributed in $[0.9,1.1]$ to prevent collisions. In (2), the ratio between $L_{i}$ and $\phi_{i}$ is from the finish tag in (1), and the scaling factor allows the choice of an appropriate scale for the virtual time in DFS. DFS, however, requires a complicated backoff interval mapping scheme to improve its throughput performance as the duration of the backoff interval is inversely proportional to the weight of a station. This mapping scheme may cause many $B I_{i}$ to be mapped to the same value, resulting in more collisions. Worse, the backoff process itself introduces randomness, causing throughput and delay variability.

\subsection{P-MAC}

In P-MAC, the weighted fairness is achieved by adjusting the contention window $(\mathrm{CW})$ of each station $j$ as follows:

$$
C W_{j}=\frac{C W_{1}-1}{\phi_{j}}+1,
$$

where $C W_{j}$ and $\phi_{j}$ are the $\mathrm{CW}$ and the weight of mobile stations of class $j$, respectively, assuming the weights of all mobile stations of class 1 are $1 . C W_{1}$ is an optimal value properly selected to reflect the number of stations contending for the wireless medium such that P-MAC can maximize the aggregate throughput. P-MAC, however, suffers from high variance of throughput and delay as it also uses backoff to avoid collisions.

\subsection{DDRR}

DDRR is based on Deficit Round Robin (DRR) [12] to translate user requirements into the IFS parameter of 802.11 MAC. Each mobile station $i$ is allocated a service quantum $Q$ bits every $T_{i}$ seconds such that $\mathrm{Q} / T_{i}$ is the desired throughput. The Deficit Counter (DC) of each station $i$ (denoted as $D C_{i}$ ) is increased at a rate of $Q$ bits every $T_{i}$ seconds, and is decreased by the size of the frame when a frame is transmitted. The deficit counter is then mapped to an appropriate IFS value at each station. At time $t$, the IFS for mobile station $i$ is calculated as: 


$$
I F S_{i}=D I F S-\alpha \frac{D C_{i}(t)}{Q} * \text { random }(1.0, \beta),
$$

where $\alpha$ is a scaling factor to ensure $I F S_{i}$ fall between PIFS and DIFS, and $\beta$ is a number larger than 1. In DDRR, no backoff algorithm as in DFS and P-MAC is employed. Collisions are avoided by using a random number between 1 and $\beta$. Thus, it eliminates the throughput and delay variability suffered by DFS and P-MAC. DDRR, however, has a potential fairness problem. This happens when a station which has accumulated a high DC value (due to transmitting at a rate lower than the desired throughput) starts transmission at a high rate. As a result, the system may fail to provide the required service to other stations since DDRR maintains the deficit count locally and does not have a global clock in the system. Moreover, when the deficit counters of all stations become zero, the stations will share the link equally. This further degrades the fairness among flows. Improper settings of parameters may also cause performance problem with DDRR.

\section{PROVIDING PER-CLASS QOS MAC LAYER MECHANISMS}

To provide QoS for IEEE 802.11 MAC, the typical approach is to tune one of the three 802.11 MAC parameters, i.e., Backoff Interval (BI), Contention Window (CW), and Inter-Frame Space (IFS), based on certain fair queuing mechanisms. The BI-based and $\mathrm{CW}$-based mechanisms are designed to control the backoff process by choosing an appropriate backoff interval for different classes of traffic. The Infer-Frame Space mechanisms use a quite different approach in which different IFS values are used to control the contention behavior of frames and no backoff process is involved. Since the BI-based and CW-based mechanisms can be designed similarly (i.e., with a backoff process), in this section, we propose two perclass QoS mechanisms for IEEE 802.11e EDCA WLANs based on whether or not a backoff process is required.

\subsection{System Architecture}

In 802.11e EDCA, service differentiation is provided for different ACs, but not for different stations. Since there are multiple ACs in the same station, two system architectures can be used to implement service differentiation. Fig. 1 shows the first option, referred to as the Joint-Scheduling EDCA (JS EDCA) architecture in this paper, in which one centralized queueing discipline combined with a backoff entity is used. When a station is ready for transmitting the next frame, the scheduler will choose a frame among the four ACs' queue according a joint queueing discipline and pass it to the backoff entity. The backoff entity then determines an appropriate backoff interval according to the frame's service level to contend with other stations. Fig. 2 shows the other option, referred to as the Separate-Scheduling EDCA (SS-EDCA), in which each AC is associated with an independent backoff entity. The backoff entity of an $\mathrm{AC}$ is used not only to contend with different ACs within the same station, but also the ACs in different stations. The design purpose of using multiple backoff entities within one station is to improve the channel utilization.

SS_EDCA is superior to JS_EDCA. For example, suppose that the backoff intervals of three frames in a station are 10, 20, and 30 slots, respectively. With SS EDCA, the station can transmit the three frames within 30 backoff slots as their backoff entities count down their contention windows simultaneously. However, if JS_EDCA is adopted, the station needs to spend 60 backoff slots to transmit these three frames. Fig. 3 plots the throughput performance of SS_EDCA and JS_EDCA if only proportional fair service is provided. In this figure, the centralized queueing discipline used in JS_EDCA is SCFQ. The distributed service discipline of EDCA is based on DFS described in Sec. II, and the simulation is conducted based on ns2 for a station with four flows of different ACs. The weights of $\mathrm{AC} 0, \mathrm{AC} 1, \mathrm{AC} 2$, and $\mathrm{AC} 3$ are set to be 0.4 , $0.3,0.2$, and 0.1 , respectively. The simulation environment and parameter settings are the same as that described in Sec. IV. The result shows that while JS_EDCA can provide perfect proportional fairness service to flows of different ACs (thanks to using a centralized fair service discipline to schedule packets of different ACs), the poor throughput is unacceptable (only about $70 \%$ of the aggregate throughput of SS_EDCA). Therefore, the proposed mechanisms are all based on the architecture of SS_EDCA in this paper.

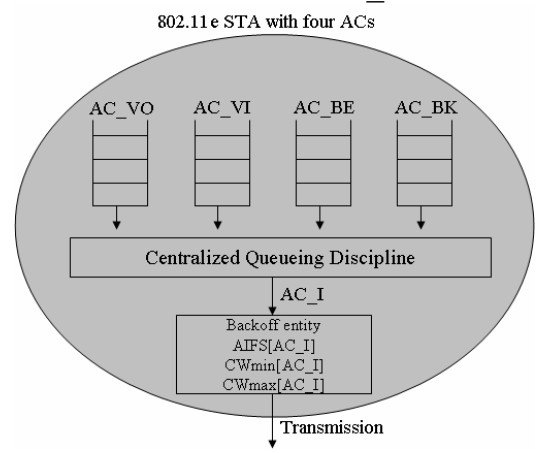

Figure 1. JS EDCA 


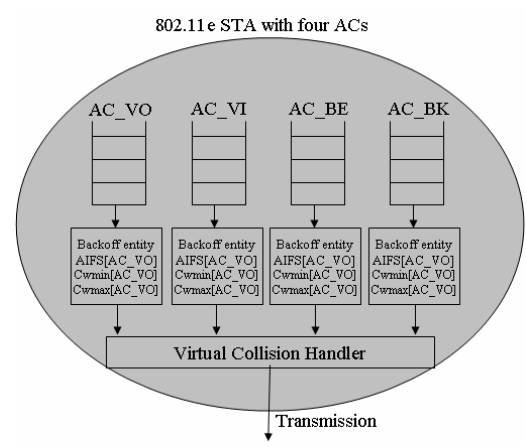

Figure 2. SS_EDCA

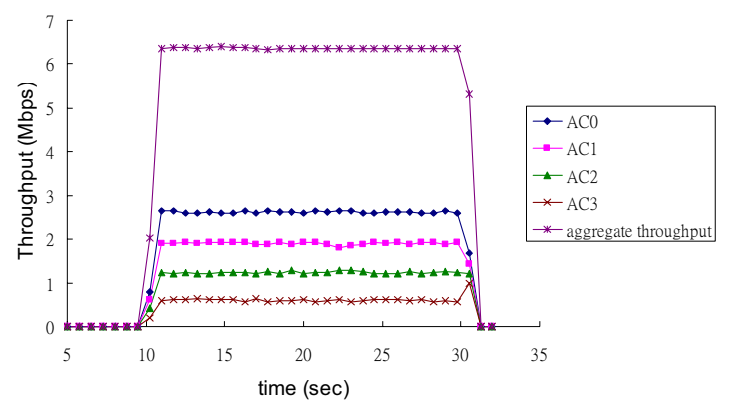

(a) SS_EDCA

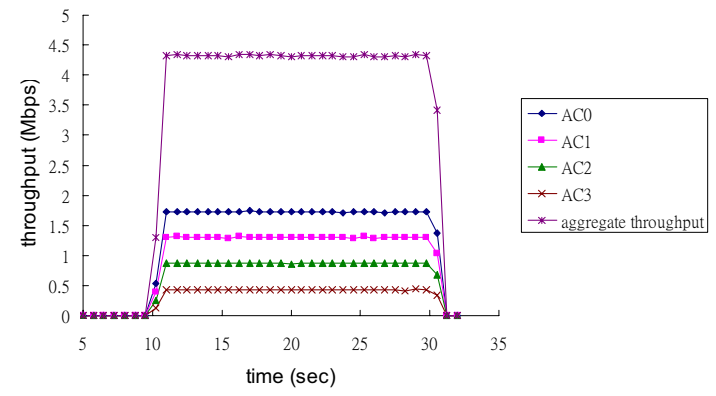

(b) JS_EDCA

Figure 3. Performance comparison of SS_EDCA and JS_EDCA

\subsection{Backoff-interval based Service Discipline}

We first describe the Backoff-Interval based Weighted Fair scheduling with Strict Priority (BIWFSP) mechanism. With BIWF-SP, 802.11e EDCA is enhanced by using Distributed Fair Scheduling (DFS) in each backoff entity so as to provide weighted fair service for flows of different ACs. The strict priority is ensured by carefully assigning the EDCA parameter set of lower priority traffic. Note that this service model can be easily extended to a contention-window based discipline if P-MAC is used to determine the backoff-interval for proportional fairness service.

\subsubsection{Proportional fairness service}

EDCA provides priority-based service differentiation for different ACs. The backoff entity of each $\mathrm{AC}$ is used to contend with different $\mathrm{ACs}$ within the same station and the ACs in different stations. Multiple backoff entities are used within one EDCA station to improve the channel utilization. This can also reduce the delay of each $\mathrm{AC}$ thanks to the eliminating of the head of line problem.

To implement proportional fairness service, each $\mathrm{AC}$ is assigned a positive real number $\phi[A C]$ indicating the weight of sharing the channel capacity. The calculation of BI (Backoff-Interval) of the head of line frame is based on (2) in DFS. Note that DFS may need a backoff interval mapping scheme to improve the throughout performance as the duration of the backoff interval is inversely proportional to the weight of a station. Any increasing function can be used as the mapping scheme of DFS, and the following squareroot mapping scheme is used in BIWF-SP.

$$
B I_{i}=\psi(\Delta)=\left\{\begin{array}{lc}
\Delta & \text { if } \Delta<\text { Threshold } \\
\lfloor\sqrt{\text { Threshold } \times \Delta}\rfloor & \text { otherwise }
\end{array}\right.
$$

where $\Delta$ denotes the backoff interval obtained in (2) and Threshold is a constant parameter.

\subsubsection{Strict priority service}

To provide strict priority, frames with higher priority must be transmitted before the lower-priority ones. In other words, the AIFS values of the lower priority must be larger than that of the higher priority plus its maximum contention window, i.e.,

$$
\operatorname{AIFS}[j]>\operatorname{AIFS}[i]+C W_{\max [i]} \text { if } j>i
$$

To sum up, in BIWF-SP, each AC is associated with a backoff entity and an EDCA parameter set, i.e., AIFS[AC], CWmin[AC], and CWmax[AC], but the value of the AIFS[AC] is now determined by (6). The backoff intervals of strict priority classes are the same as EDCA and that of proportional classes are calculated based on (2). Besides, the backoff entities of different ACs in the same station work independently. Ties are broken arbitrarily upon parallel access to the channel at the same slot. The operation is the same when different ACs in different stations are considered.

Note that while BIWF-SP can provide the desired service model, it is inefficient. When the higher priority classes are inactive, the lower priority classes still need to wait for the large AIFS before the backoff interval is counted down to zero. Therefore, the aggregate throughput will degrade. Moreover, the Scaling_Factor of DFS needs to be tuned according to the traffic load. This also introduces uncertainty in the performance of BIWF-SP. 


\subsection{IFS-based Service Discipline}

Next, we propose the IFS-based Distributed Fair Queuing with Strict Priority (IDFQ-SP) mechanism. IDFQ-SP chooses an appropriate IFS value in proportion to the finish tag of the frame to be transmitted. Note that the description and simulations of IDFQ-SP in this paper is based on the parameters of 802.11 b. It can be easily extended to other 802.11 variations (e.g., $802.11 \mathrm{a} / \mathrm{g}$ ).

\subsubsection{Proportional fairness service}

To provide proportional service, we emulate SCFQ in a distributed manner. Each head of line frame is stamped with a finish tag. The QoS metrics translated from user requirements are then mapped to the IFS of 802.11 DCF. A smaller finish tag is mapped to a smaller IFS value, and thus the frame with the smallest tag is transmitted first. There is no backoff mechanism in IDFQ-SP, and hence no throughput fluctuation problem as in DFS and P-MAC. The collisions are avoided by randomizing each IFS. Moreover, the proposed mechanism eliminates the requirement of backoff mapping schemes as in DFS. The aggregate throughput is improved by better design of IFS. Finally, IDFQ-SP is designed to be scalable, thus it works efficiently even in a wireless network with many nodes.

In IDFQ-SP, each backoff entity $i$ maintains a local virtual clock $v_{i}(t)$, where $v_{i}(0)=0$. Each transmitted frame is stamped with a finish tag. The calculation of the tag is also based on (1), except that the tag is calculated when the frame is at the head of line, instead of at arrival to the system. The virtual clock is updated when backoff entity $i$ transmits or hears ${ }^{1}$ a data or ACK frame with finish tag $F$, say, at time $t$, and $v_{i}(t)=\max \left\{v_{i}(t), F\right\}$. As such, the virtual clock is ensured to grow monotonically as frames with larger finish tags may be transmitted before those with smallest finish tag due to randomization adopted in IDFQ-SP described below. Note that since all mobile stations are associated with the same access point, their local virtual clocks of the backoff entities should be equal. Therefore, the global clock in the system can be maintained.

The finish tag is then mapped to the IFS parameter of 802.11. A smaller finish tag is mapped to a smaller IFS value, so that the frame with the smallest tag could

\footnotetext{
${ }^{1}$ The access point or mobile stations will attach the finish tag of the received frame on the ACK frame to help to maintain the system virtual clock
}

be transmitted first due to the smallest waiting time. To be compatible with the 802.11 MAC protocol, IFS is maintained in a range between PIFS (e.g., 30us in $802.11 \mathrm{~b}$ ) and DIFS (50us in 802.11b). Let $F_{i}$ be the finish tag of the head of line frame for backoff entity $i$, and $L_{\max }$ and $\phi_{\min }$ be the maximum frame size and minimum weight in the system, respectively. The IFS of backoff entity $i$ is derived as

$$
I F S_{i}= \begin{cases}40(\mu s)+\frac{\text { random }(0,1)}{\alpha} \times \beta, & \text { if } F_{i}-v_{i}(t)=0 \\ 40(\mu s)+\frac{F_{i}-v_{i}(t)}{\alpha} \times \beta, & \text { otherwise }\end{cases}
$$

where $\alpha=L_{\max } / \phi_{\min }$, which is the maximum value of $F_{i}-v_{i}(t)$; the positive random number $\beta$ is introduced to prevent collisions.

Note that while the frame with the smallest finish tag is usually transmitted when the network is ready for service, a frame with a larger finish tag may sometimes be transmitted earlier as we use a random factor $\beta$ to avoid collision in the distributed environment. When this happens, the value of $F_{i}-v_{i}(t)$ may be less than 0 . Even so, the value of $\left|F_{i}-v_{i}(t)\right|$ is less than or equal to $\alpha$. Consequently, $\left(F_{i}-v_{i}(t)\right) / \alpha$ stays between -1 and 1 . Moreover, if $\beta$ is uniformly distributed over the interval $[0,10], I F S_{i}$ is bounded between PIFS and DIFS, ensuring IDFQSP to be backwards compatible with IEEE 802.11.

\subsubsection{Strict priority service}

To provide strict priority service, disjoint intervals between PIFS and DIFS are reserved for the AIFSs of high priority $\mathrm{ACs}$, i.e., $\mathrm{AC}[0]$ and $\mathrm{AC}[1]$. These intervals must be less than those used for proportional sharing and cannot be used for proportional fair service. To achieve this purpose, we can limit $\beta$ in (7) to a value uniformly distributed over the interval $[0,5]$ and assign the AIFSs of the frames of AC[0] and AC[1] as: PIFS + uniform $(0,2.5)$ and $\operatorname{PIFS}+$ uniform $(2.5,5)$, respectively.

To sum up, there is no backoff mechanism in IDFQ-SP. The AIFSs of the strict priority classes are their PIFS plus a disjoint interval and those of proportional classes are calculated based on (7). Besides, the system virtual clock is used only for proportional service classes. Therefore, the frames of the strict priority classes will not attach its finish tags 
on the frames such that the virtual clocks of other backoff entities will not be updated.

\section{PERFORMANCE EVALUATION}

In this section, we evaluate the performance of the proposed mechanisms via simulation based on ns-2 EDCA implementation created by Chesson and Singla [13]. The wireless link rate is $11 \mathrm{Mbps}$ and each data flow is associated with one $\mathrm{AC}$ and generates CBR UDP traffic at a rate of $8 \mathrm{Mbps}$ to the AP. The packets, including the IP header, of all ACs are fixed at 1500 bytes. Both $\mathrm{AC}[0]$ and $\mathrm{AC}[1]$ provide strict priority with $\mathrm{AC}[0]>\mathrm{AC}[1]$; both $\mathrm{AC}[2]$ and $\mathrm{AC}[3]$ provide weighted fair service with weights of $2: 1$. The parameters Scaling_Factor and Threshold of DFS are set to 0.01 and 80 , respectively, in the simulations for BIWF-SP. The values of the EDCA parameter sets for the four ACs are listed in Table 1.

Table 1. EDCA parameter sets used in the simulations

\begin{tabular}{|l|c|c|c|c|}
\hline & $\mathrm{AC}$ VO & $\mathrm{AC}$ VI & $\mathrm{AC} \_\mathrm{BE}$ & $\mathrm{AC} \_\mathrm{BK}$ \\
\hline$C W \min$ & 3 & 7 & 15 & 15 \\
\hline$C W \max$ & 7 & 15 & 1023 & 1023 \\
\hline
\end{tabular}

We first demonstrate the effect of strict priority implemented in our mechanisms for delay-sensitive traffic as compared to the original EDCA. Fig. 4 plots the average delay and delay variation of $\mathrm{AC}[0]$ as a function of the number of $\mathrm{AC}[1]$ flows. In this simulation, only $\mathrm{AC}[0]$ and $\mathrm{AC}[1]$ flows are active. It shows that the original EDCA is very sensitive to the number of active low priority flows, while in both BIWF-SP and IDFQ-SP, the average delays and delay variations are invariant to the traffic load of active low priority. This proves that the two proposed mechanisms are suitable to provide service for delay sensitive traffic such as VoIP over WLANs.

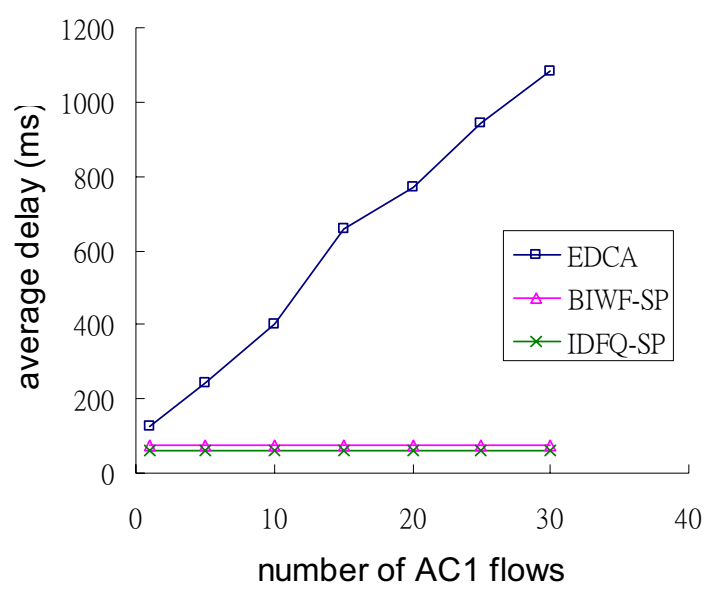

(a) Average delay of $\mathrm{ACO}$

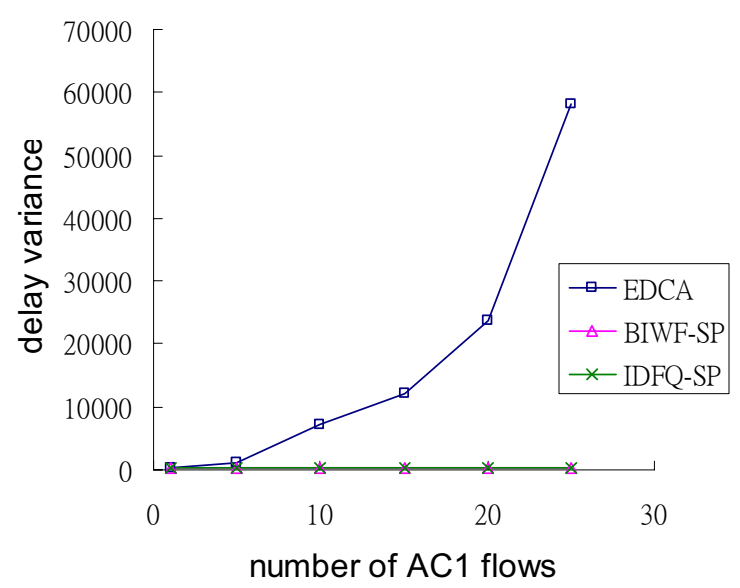

(b) Delay variance of $\mathrm{ACO}$

Figure 4. The delay and inter-arrival time of flows

Next, we compare the throughput performance of the original EDCA, BIWF-SP and IDFQ-SP, in terms of providing both strict priority and proportional service. We consider three different scenarios. The first scenario considers four different AC flows within the same station (STA) (Fig. 5(a)); the second scenario shows the case of distributing four different AC flows to four different stations (Fig. 5(b)); the third scenario depicts the case that not all ACs in one station are active (Fig. 5(c)). In this simulation, the sending rates of all flows of each $\mathrm{AC}$ are initialized at $8 \mathrm{Mps}$. The rates of $\mathrm{AC}[0]$ 's flows are changed to $5 \mathrm{Mbps}, 4 \mathrm{Mbps}$, and $3 \mathrm{Mbps}$ at times $15 \mathrm{sec}, 20 \mathrm{sec}$, and $25 \mathrm{sec}$, respectively; those of $\mathrm{AC}[1]$ 's flows are changed to $2 \mathrm{Mbps}$ and $1 \mathrm{Mbps}$ at times $20 \mathrm{sec}$ and $25 \mathrm{sec}$, respectively. Note that due to space limitations, we only provide the results of scenario 1 in this paper. The results of other scenarios are consistent with Fig. 6.

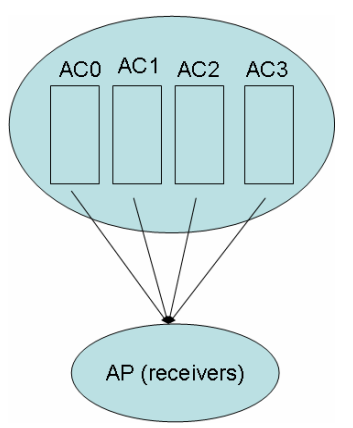

(a) 


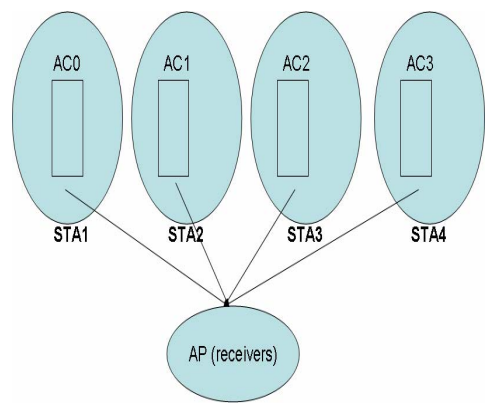

(b)

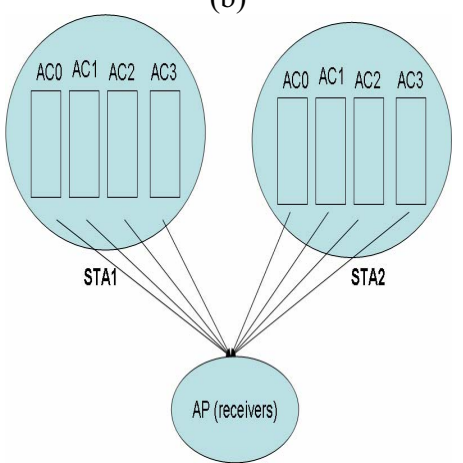

(c)

Figure 5. Simulation scenarios

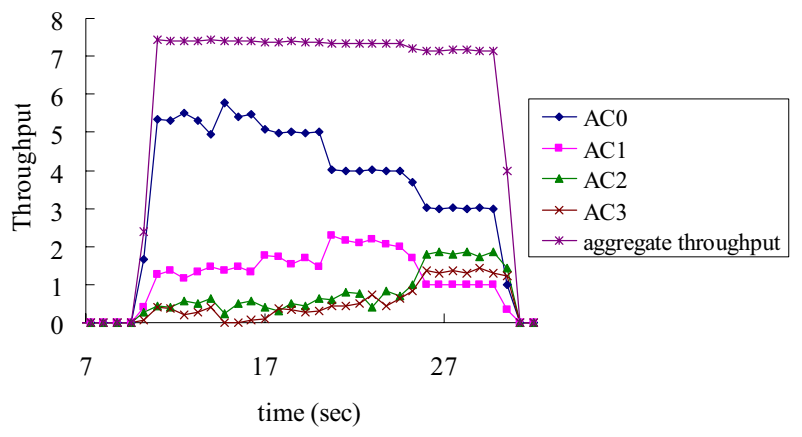

(a) EDCA

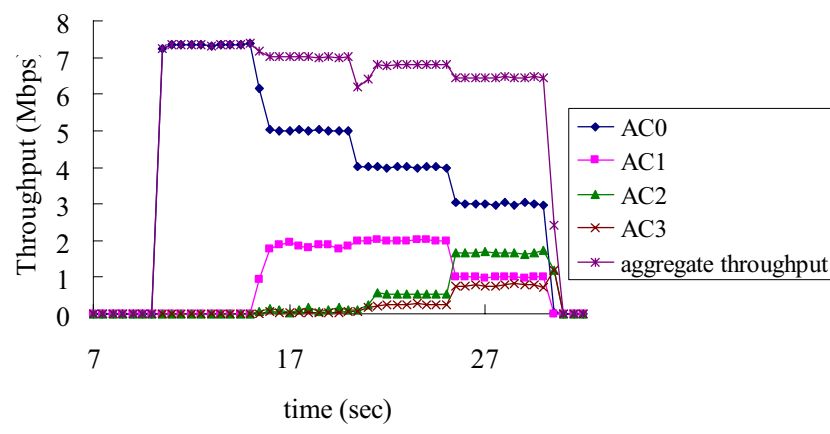

(b) BIWF-SP

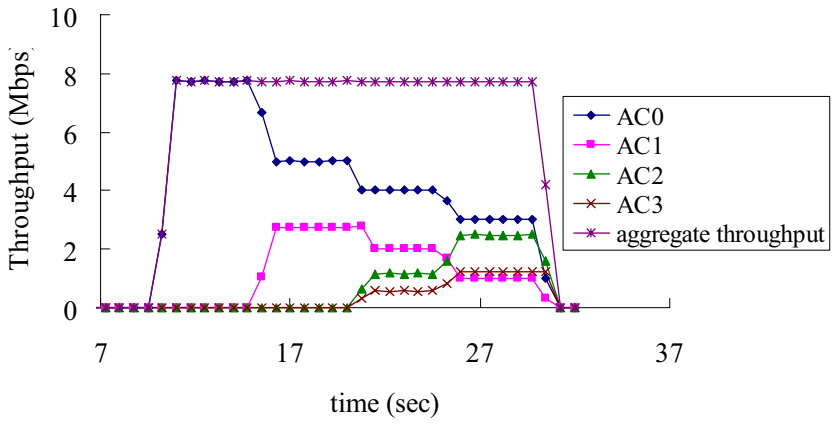

(c) IDFQ-SP

Figure 6 . The throughputs of three schemes

Fig. 6 plots the throughputs of the original EDCA, BIWF-SP, and IDFQ-SP. the results show that while EDCA provides priority-based service, it is still not a strict priority. With EDCA, the performance of $\mathrm{AC}[0]$ degrade when traffic of the highest priority $\mathrm{AC}[1]$ is active, and the flows of $\mathrm{AC}[2]$ and $\mathrm{AC}[3]$ can not be allocated resource in proportion to their assigned weights. BIWF-SP and IDFQ-SP, on the other hand, can ensure both strict priority and proportional fairness service for flows of each AC such that only when the aggregate sending rate of $\mathrm{AC}[0]$ and $\mathrm{AC}[1]$ is less than the link capacity, $\mathrm{AC}[2]$ and $\mathrm{AC}[3]$ can receive services, and the resource is allocated proportionally. Compared to BIWF-SP, IDFQ-SP is more immune to the impact of higher priority flows, and has higher aggregate throughput when the sending rates of higher priority flows decrease. Besides, IDFQ-SP is more stable than BIWF-SP, rendering it an excellent candidate to provide per-class QoS service at the MAC layer for IEEE 802.11 LANs.

\section{CONCLUSION}

In this paper, we propose two mechanisms, called BIWF-SP and IDFQ-SP, based on backoff interval (BI) and inter-frame space (IFS), respectively, to provide per-class QoS at the MAC layer for IEEE 802.11e EDCA WLANs. In our mechanisms, both strict priority and proportional fair service differentiation among different service classes are supported. We describe the operation in details, and compare their performance with the original EDCA mechanism via simulation. The simulation results show that both BIWF-SP and IDFQ-SP outperform the original EDCA in terms of providing support to both strict priority and weighted fair service. Compared to IDFQ-SP, BIWF$\mathrm{SP}$ is easier to implement in real systems; compared to BIWF-SP, IDFQ-SP has better aggregate throughput and is more stable. More importantly, both mechanisms conform with the IEEE 802.11e EDCA 
standard, rendering both good candidates to provide per-class QoS service for IEEE 802.11 WLANs.

\section{ACKNOWLEDGEMENTS}

This paper was supported in part by National Science Council (NSC), Taiwan, under a Center Excellence Grant NSC93-2752-E-002-006-PAE, and in part by NSC under Grant Number NSC93-2213-E-002-132.

\section{REFERENCE}

[1] IEEE 802.11, Wireless LAN Medium Access Control (MAC) and Physical Layer (PHY) specifications, IEEE Standard, Aug. 1999.

[2] I. Aad and C. Castelluccia, "Differentiation Mechanisms for IEEE," IEEE INFOCOM 2001.

[3] J. Deng and R.S. Chang, "A Priority Scheme for IEEE 802.11 DCF Access Method," IEICE Trans. Commun., Vol. E82-B, no. 1, 1999, pp. 96-102

[4] V. Kanodia, C. Li, A. Sabharwal, B. Sadeghi and E. Knightly, "Distributed Multi-Hop Scheduling and Medium Access with Delay and Throughput Constraints," ACM Mobicom 2001

[5]S. Golestani, “A Self-Clocked Fair Queueing Scheme for Broadband Applications." IEEE INFOCOM'94, page 636-646, Toronto, CA, Jane 1994

[6] S. Lu, T. Nandagopal, and V. Bharghavan, "A Wireless Fair Service Algorithm for Packet Cellular Networks," ACM Mobicom 1998.

[7] N. H. Vaidya, P. Bahl, and S. Gupta, "Distributed Fair Scheduling in Wireless LAN," ACM Mobicom 2000.

[8] D. Qiao and K. G. Shin, “Achieving Efficient Channel Utilization and Weighted Fairness for Data Communications in IEEE 802.11 WALN under the DCF," ACM IWQoS 2002.

[9] W. Pattara-Atikom, S. Banerjee, and P. Krishnamurthy, "Starvation Prevention and Quality of Service in Wireless LANs." IEEE WPMC 2002.

[10] IEEE WG, Draft Supplement to Standard for Telecommunications and Information Exchange between Systems-LAN/MAN Specific Requirements- Part 11: Wireless LAN Medium Access Control (MAC) and Physical Layer (PHY) Specifications: Medium Access Control (MAC), Enhancements for Quality of Service (QoS), IEEE 802.11e Draft 4.1, Feb. 2003.
[11] M. Malli, Q. Ni, T. Turletti and C. Barakat, "Adaptive Fair Channel Allocation for QoS Enhancement in IEEE 802.11 Wireless LANs.” IEEE ICC 2004.

[12] M. Shreedhar and G. Varghese, "Efficient Fair Queuing Using Deficit Round-robin," IEEE/ACM Trans. Net., vol. 4, no. 3, 1996, pp. 375-85.

[13] ns-2, available at http://www.isi.edu/nsnam/ns/ 